\title{
Colouring Multijoints
}

\author{
Anthony Carbery • Stefán Ingi Valdimarsson
}

Received: 6 September 2013 / Revised: 28 May 2014 / Accepted: 5 July 2014 /

Published online: 21 August 2014

(C) Springer Science+Business Media New York 2014

\begin{abstract}
Let $\mathbb{F}$ be a field, let $L_{1}, \ldots, L_{d}$ be pairwise disjoint collections of lines in $\mathbb{F}^{d}$, and let $\mathcal{L}=\left\{L_{1}, \ldots, L_{d}\right\}$. We say that a point $x \in \mathbb{F}^{d}$ is a multijoint of $\mathcal{L}$ if $x$ lies on a line from each of the collections in $\mathcal{L}$, and moreover the directions of these lines span $\mathbb{F}^{d}$. We prove that there exists a constant $C_{d}$ such that if $\mathcal{L}$ is a generic family of collections of lines in $\mathbb{F}^{d}$ and $J$ is a set of multijoints of $\mathcal{L}$, then there exists a $d$-colouring $\kappa: J \rightarrow\{1,2, \ldots, d\}$ such that for each $j$, for each $l \in L_{j}$ we have $|\{x \in J \cap l: \kappa(x)=j\}| \leq C_{d}|J|^{1 / d}$.
\end{abstract}

Keywords Multijoints · Colourings $\cdot$ Multilinear Kakeya

\section{Introduction}

Let $\mathbb{F}$ be a field, let $L_{1}, \ldots, L_{d}$ be pairwise disjoint collections of lines in $\mathbb{F}^{d}$, and let $\mathcal{L}=\left\{L_{1}, \ldots, L_{d}\right\}$. We say that a point $x \in \mathbb{F}^{d}$ is a multijoint of $\mathcal{L}$ if $x$ lies on a line from each of the collections in $\mathcal{L}$, and moreover the directions of these lines span $\mathbb{F}^{d}$.

Regard each line of $L_{j}$ as being coloured with colour $j$. In this note we address the problem of colouring the set $J$ of multijoints of $\mathcal{L}$ with as few colours as possible in such a way that no line of a given colour contains too many points of that same colour. We need to make these notions precise, and do so in the statement of our main result. Further clarification and a discussion of the context of the result follows in the remarks after its statement. The family $\mathcal{L}$ is said to be generic if whenever $l_{j} \in L_{j}$ meet at $x$, then the directions of the $l_{j}$ span $\mathbb{F}^{d}$.

\section{A. Carbery}

School of Mathematics and Maxwell Institute for Mathematical Sciences, University of Edinburgh, JCMB, King's Buildings, Mayfield Road, Edinburgh EH9 3JZ, Scotland, UK e-mail: a.carbery@ed.ac.uk 
Theorem 1 Let $\mathcal{L}$ be a generic family of collections of lines in $\mathbb{F}^{d}$ as above, and let $J$ be a (finite) set of multijoints of $\mathcal{L}$. Then there exists a constant $C_{d}$ which depends only on the dimension $d$ and not on $\mathcal{L}$ or $J$, and a $d$-colouring $\kappa: J \rightarrow\{1,2, \ldots, d\}$ such that for each $j$, for each $l \in L_{j}$,

$$
|\{x \in J \cap l: \kappa(x)=j\}| \leq C_{d}|J|^{1 / d} .
$$

Remark 1 We emphasise that while an $x \in J$ can arise as a multijoint in several ways (i.e. there may be many $d$-tuples of lines $\left(l_{1}, \ldots, l_{d}\right) \in \mathcal{L}_{1} \times \cdots \times \mathcal{L}_{d}$ with $x \in l_{j}$ for all $j$ ) we only associate a single colour to it. That is, a multijoint is a point of $\mathbb{F}^{d}$, not a $d$-tuple of lines.

Remark 2 Matters are trivial if we allow more than $d$ colours for $J$ : simply colour every point of $J$ with colour $d+1$. We therefore consider $d$-colourings $\kappa: J \rightarrow\{1,2, \ldots, d\}$ of $J$.

Remark 3 We cannot hope for each line of a given colour to contain at most about $|J|^{\beta}$ points of $J$ of the same colour unless $\beta \geq 1 / d$. To see this consider the monkeybar/jungle-gym example where $L_{j}$ consists of $N^{d-1}$ lines parallel to the $x_{j}$-axis passing through the points $\left(m_{1}, \ldots, m_{j-1}, 0, m_{j+1}, \ldots, m_{d}\right)$ for $m_{i} \in\{1, \ldots, N\}$. If each line of $L_{j}$ contains at most $K$ multijoints of colour $j$ then there are at most $N^{d-1} K$ multijoints of colour $j$ altogether and hence at most $d N^{d-1} K$ multijoints altogether. But there are $N^{d}$ multijoints in this example, so we must have $N^{d} \leq d N^{d-1} K$. Hence $K$ must satisfy $K \geq N / d=|J|^{1 / d} / d$.

Remark 4 We cannot expect in general to use fewer than $d$ colours. To illustrate this in the case $d=3$, put $2 N$ red lines parallel to $e_{1}$ passing through the points $(0, j, 0)$ for $1 \leq j \leq N$ and $(0,0, j)$ for $1 \leq j \leq N$, and similarly put $2 N$ blue lines parallel to $e_{2}$ and $2 N$ green lines parallel to $e_{3}$ in the corresponding places. Then on the plane $x_{3}=0$ we have an $N \times N$ square lattice of $N$ red lines parallel to $e_{1}$ and $N$ blue lines parallel to $e_{2}$. Through each lattice point on this plane put a green line to make it a multijoint in $J$ but in such a way that no new multijoints in $J$ are created. Similarly add red lines through lattice points on the plane $x_{2}=0$ and blue lines through lattice points on the plane $x_{1}=0$. Altogether we now have $3 N^{2}$ multijoints, with the colours red, blue and green in symmetry. Can we colour this arrangement of multijoints using only two colours, say red and blue, in such a way that a line of a given colour contains at most $\sim N^{2 / 3}$ points of that colour? If so, considering the multijoints in the the plane $x_{3}=0$, every red line would have at most $\sim N^{2 / 3}$ red multijoints, so there would be at most $\sim N \times N^{2 / 3}=N^{5 / 3}$ red multijoints on this plane, and simlarly at most $\sim N^{5 / 3}$ blue multijoints. Hence there would be at most $\sim N^{5 / 3}$ multijoints on this plane, when in fact there are $\sim N^{2}$. This contradiction shows that we cannot colour this arrangement with fewer than three colours, and similar examples in higher dimensions show that in $\mathbb{F}^{d}$ we will need $d$ colours in general.

Our setting with $d$ families of lines is a variant of the setting of the so-called joints problem. There we have a single collection $L$ of lines in $\mathbb{F}^{d}$, and we define a joint of $L$ 
to be any point which lies at the intersection of $d$ lines from $L$ with the condition that the set of directions of those $d$ lines should span $\mathbb{F}^{d}$. In recent years there has been quite a bit of interest in the joints problem and it is now known that if $J$ is the set of joints of $L$ then we have

$$
|J| \leq C_{d}|L|^{d /(d-1)}
$$

where $C_{d}$ depends only on the dimension $d$. This was originally proved in the case $\mathbb{F}=\mathbb{R}$ by Guth and Katz in [7] for $d=3$, then for a general $d \geq 3$ by Quilodrán [10] and independently by Kaplan, Sharir and Shustin [9]. Treatments of the extension to general fields are in [2,5] and [11].

Let us temporarily pass to the situation $\mathbb{F}=\mathbb{R}$. Let $N_{1}, \ldots, N_{d}$ be natural numbers and consider the grid $\mathcal{G}$ of points $\left\{1,2, \ldots, N_{1}\right\} \times \cdots \times\left\{1,2, \ldots, N_{d}\right\} \subseteq \mathbb{R}^{d}$. Let us consider the family $L_{j}$ of $N_{1} N_{2} \cdots N_{j-1} N_{j+1} \cdots N_{d}$ lines parallel to the $j$ th coordinate axes passing through the points of the grid $\mathcal{G}$. Then it is easy to see that $|J|=N_{1} \cdots N_{d}$ while $\prod_{j=1}^{d}\left|L_{j}\right|=\left(N_{1} \cdots N_{d}\right)^{d-1}$. This example therefore gives rise to the natural question in our setting as to whether we have, with $J$ now being the set of multijoints of $\mathcal{L}$,

$$
|J| \leq C_{d} \prod_{j=1}^{d}\left|L_{j}\right|^{1 /(d-1)}
$$

where $C_{d}$ depends only on the dimension $d$. At the moment this question seems difficult (except in two dimensions, in which case it is obvious) ${ }^{1}$ and we instead consider the related problem described above.

Let us explain the relevance of our result to inequality (2). In [4] Dvir proved the finite field Kakeya conjecture. Since then, his central idea, dubbed the polynomial method, has been used extensively, among other things in the cited work on the joints problem. In another direction, Guth [6] extended the polynomial method to prove the endpoint case of the multilinear Kakeya conjecture in $\mathbb{R}^{d}$. This is a continuous version of inequality (2) introduced above. His proof used algebraic topology but see [3] for a treatment which relies only on the Borsuk-Ulam theorem.

Suppose we have $d$ families $\mathcal{T}_{j}$ of doubly-infinite tubes $T_{j}$ in $\mathbb{R}^{d}$ of infinite length and unit cross-section, and suppose that each tube in $\mathcal{T}_{j}$ points approximately in the direction of the $j$-th standard basis vector $e_{j} .{ }^{2}$ The main substance of Guth's argument consists of proving that for every non-negative function $M$ there exist functions $S_{j}$, $j=1, \ldots, d$, such that

$$
M(x) \leq \prod_{j=1}^{d} S_{j}(x)^{1 / d}
$$

\footnotetext{
1 However, Iliopoulou [8] has recently informed us that she has established (2) when $\mathbb{F}=\mathbb{R}$ for arbitrary $d$ and also for arbitrary $\mathbb{F}$ when $d=3$. In neither case is the genericity hypothesis needed.

2 Guth's set up is more relaxed than this, see also [1].
} 
and

$$
\sum_{x} S_{j}(x) \leq C_{d}\|M\|_{d}
$$

The domain of the functions $M$ and $S_{j}$ is the set of unit cubes in $\mathbb{R}^{d}$, and inequality (3) is supposed to hold for each cube. The sum in inequality (4) is over cubes $x$ meeting a tube $T_{j} \in \mathcal{T}_{j}$, and inequality (4) is supposed to hold for each tube in the collection $\mathcal{T}_{j}$, for all $j=1, \ldots, d$. Loosely speaking, Guth's functions $S_{j}(x)$ are constructed geometrically as the directional surface areas of $Z_{p} \cap x$ where $Z_{p}$ is the zero set of a polynomial $p$ of degree dominated by $\|M\|_{d}$ which bisects each cube $x$ in the domain of $M$ in a particularly strong way.

In considering inequality (2) one is naturally led to consider inequalities (3) and (4) where the tubes are replaced by lines (of zero width), the unit cubes by points and where we suppose that if $x \in l_{j} \in L_{j}$ for $j=1, \ldots, d$ then the directions of the $l_{j}$ should span $\mathbb{F}^{d}$, i.e. that $x$ is a multijoint according to our definition above. Note that in the case of $\mathbb{R}^{d}$ straightforward limiting arguments applied to the results of [6] and [1] do not yield an answer to the question of the satisfiability of (3) and (4), or of the validity of (2) in this setting. Note also that there is no obvious analogue of the notion of directional surface area in our discrete setting.

If in (3) and (4) we replace the general nonnegative function $M$ by a characteristic function $\chi_{J}$ of a set of multijoints $J$, and the geometric mean by the (larger) arithmetic mean we arrive at the (easier) problem of finding $S_{j}$ such that

$$
\chi_{J}(x) \leq \frac{1}{d} \sum_{j=1}^{d} S_{j}(x)
$$

and

$$
\sum_{x} S_{j}(x) \leq C_{d}|J|^{1 / d}
$$

Theorem 1 is equivalent to this new problem: if we have such $S_{j}$, for each $x$, choose a $j$ with $S_{j}(x) \geq 1$ and assign colour $j$ to $x$; conversely, if we have a colouring satisfying the conclusion of Theorem 1, declare $S_{j}(x)=d$ if $x$ has colour $j$ and $S_{j}(x)=0$ otherwise. (See also Remark 7 at the end of the note.)

We now make some remarks on the relation between our colouring result and the joints theorem (1). In the first place, our result implies a weak form of the joints theorem in so far as if we have a generic family of lines $\left\{L_{1}, \ldots, L_{d}\right\}$ our result enables us to estimate the size of its set of multijoints $J$ as

$$
\begin{aligned}
|J| & =\sum_{x \in J} 1 \leq C_{d} \sum_{x \in J} \sum_{j=1}^{d} S_{j}(x)=C_{d} \sum_{j=1}^{d} \sum_{x \in J} S_{j}(x) \\
& \leq C_{d} \sum_{j=1}^{d} \sum_{l_{j} \in L_{j}} \sum_{x \in J \cap l_{j}} S_{j}(x) \leq C_{d} \sum_{j=1}^{d}\left|L_{j}\right||J|^{1 / d}
\end{aligned}
$$


from which it follows that $|J| \leq C_{d}\left(\sum_{j=1}^{d}\left|L_{j}\right|\right)^{d /(d-1)}$. Secondly, our argument uses important aspects of the arguments used to prove the joints theorem. In particular it makes use of a fundamental lemma of Quilodrán from [10] (see Lemma 1 below) and indeed the strategy of his original proof of the joints theorem. Quilodrán's lemma is proved using the polynomial method and so there is a polynomial underlying our own colouring theorem; however, due to the iterative nature of our argument it is not clear that one can express the quantities $S_{j}$ directly in terms of the zero set of a suitable polynomial.

In this direction, one strategy for proving our theorem might be as follows: choose a polynomial $p$ of degree dominated by $|J|^{1 / d}$ which vanishes at each member of $J$. (One can do this simply by observing that if deg $p \gtrsim_{d}|J|^{1 / d}$ one can choose the coefficients of $p$ such that it satisfies the $|J|$ linear equations $p(x)=0, x \in J$.) Then, if $x$ is a multijoint such that $\nabla p(x) \neq 0$, it must be the case that for some $j$, for all $l_{j} \in L_{j}$, we have $l_{j} \nsubseteq Z_{p}$. (Otherwise, for all $j$ there exists an $l_{j} \in L_{j}$ such that $p$ vanishes identically on $l_{j}$, and thus its directional derivative $\omega_{l_{j}} \cdot \nabla p$ also vanishes on $l_{j}$; but for $x \in \cap_{j} l_{j}$ we have that $\nabla p(x)$ is a linear combination of $\omega_{l_{j}} \cdot \nabla p(x)$ since the directions $\omega_{l_{j}}$ span $\mathbb{F}^{d}$.) If so, choose the colour $\kappa(x)$ of $x$ to be $j$ and observe that if $l_{j} \in L_{j}$ then $\left\{x \in l_{j} \cap J: \kappa(x)=j\right.$ and $\left.\nabla p(x) \neq 0\right\} \subseteq l_{j} \cap Z_{p}$ which has cardinality at most deg $p \leq C_{d}|J|^{1 / d}$. However this argument does not deal with any critical points of $p$ and for this reason we are forced to adopt an alternative strategy as below.

Finally, we remark that when $d=2$ there is a simple ad hoc argument leading to the conclusion of Theorem 1 . Indeed, suppose in $\mathbb{F}^{2}$ we have a family of blue lines and a family of red lines (with no line having both colours). If a blue line contains at most $\sqrt{2}|J|^{1 / 2}$ bijoints, colour all of those bijoints blue. Colour all other bijoints red. Suppose we have a red line with as many as $k=\sqrt{2}|J|^{1 / 2}+1$ red bijoints on it. Then each of these bijoints is on a (different) blue line, which must therefore contain more than $\sqrt{2}|J|^{1 / 2}$ bijoints (as they are not all blue). Hence there are more than

$$
\begin{aligned}
& \sqrt{2}|J|^{1 / 2}+\left(\sqrt{2}|J|^{1 / 2}-1\right)+\left(\sqrt{2}|J|^{1 / 2}-2\right)+\cdots+\left(\sqrt{2}|J|^{1 / 2}-(k-1)\right) \\
& =\sqrt{2} k|J|^{1 / 2}-(k-1) k / 2=\sqrt{2}\left(\sqrt{2}|J|^{1 / 2}+1\right)|J|^{1 / 2}-\sqrt{2}|J|^{1 / 2}\left(\sqrt{2}|J|^{1 / 2}+1\right) / 2 \\
& =|J|+\frac{|J|^{1 / 2}}{\sqrt{2}}>|J|
\end{aligned}
$$

distinct bijoints altogether, which is a contradiction. Hence each red line also contains at most $\sqrt{2}|J|^{1 / 2}$ red points too.

\section{Proof of Theorem 1}

We let $m$ be a positive integer and $J$ be a set of multijoints of $\mathcal{L}$. We say that a colouring $\kappa: J \rightarrow\{1, \ldots, d\}$ is $m$-bounded if for any $j=1, \ldots, d$ and any $l_{j} \in L_{j}$ we have

$$
\left|\left\{x \in l_{j}: \kappa(x)=j\right\}\right| \leq m .
$$


Otherwise the colouring will be called $m$-unbounded. So in an $m$-unbounded colouring there is some line containing $(m+1)$ members of $J$ with the same colour as the line. Theorem 1 can be restated as:

Theorem 2 Let $\mathcal{L}$ be a generic family of collections of lines in $\mathbb{F}^{d}$ as above and let $J$ be a set of multijoints of $\mathcal{L}$. Then there exists a constant $C_{d}$ which depends only on the dimension $d$ and not on $\mathcal{L}$ or $J$ and an integer $m$ with $m \leq C_{d}|J|^{1 / d}$ such that $J$ is colourable with an $m$-bounded colouring.

Proof Fix a positive integer $m$ and the set $\mathcal{L}$. Let $J_{c}$ be a set of multijoints of $\mathcal{L}$ which is colourable with an $m$-bounded colouring and let $x_{0}$ be a multijoint of $\mathcal{L}$ which does not belong to $J_{c}$.

Our aim is to prove the following claim.

Claim 1 If $m>C_{d}\left|J_{c}\right|^{1 / d}$ then $\tilde{J}=J_{c} \cup\left\{x_{0}\right\}$ is colourable with an $m$-boundeded colouring.

This claim immediately proves the theorem: every singleton subset of $J$ is trivially colourable with an $m$-bounded colouring and the claim allows us to add points one at a time, preserving the property of being colourable with an $m$-bounded colouring, until the size of the set reaches $\left(m / C_{d}\right)^{d}$, which by assumption will not happen before we exhaust $J .^{3}$

We now turn to the proof of the claim. To simplify notation we use $J$ for what is called $J_{c}$ in the statement of the claim. Let us label the elements of $J=\left\{x_{1}, \ldots, x_{v}\right\}$ and order the multijoints in $\tilde{J}$ according to the indices. Let $K$ be the set of colourings of $J$ which are $m$-bounded.

We wish to define a strict partial ordering on $K$. To do this we construct for every $\kappa \in K$ a coloured rooted tree $T$ whose vertices belong to $\tilde{J}$. The tree will be rooted at $x_{0}$ (which is achromatic) and all the other vertices will be members of $J$ and will be assigned the colour given to them by $\kappa$. (We shall not colour the edges of the tree.)

\subsection{Construction of the Tree}

Let us fix a $\kappa \in K$ and describe the construction of the tree $T$ with an iterative process.

At the 0 -th step, the tree $T_{0}$ has one vertex, $x_{0}$, and no edges. We will maintain an ordering on the vertices, based primarily on the step in which a vertex gets added and secondarily on the ordering inherited from $\tilde{J}$. In accordance with that we say that $x_{0}$ is the first element of the tree and give it the alternative name $y_{1}$.

At the $i$-th step we consider $T_{i-1}$, and either construct a $T_{i}$, or else stop the procedure and declare $T:=T_{i-1}$. We consider the $i$-th element of the tree $T_{i-1}$, which we call $y_{i}$, and construct $T_{i}$ by adding one or more children from amongst the members of $\tilde{J}$ not already in $T_{i-1}$ to $T_{i}$, and then connect $y_{i}$ to its children with edges.

If there is no $i$-th element in the tree $T_{i-1}$ we say that the tree is fully constructed and we define $T:=T_{i-1}$. Clearly this must happen before or when we reach step $|J|$.

\footnotetext{
${ }^{3}$ As we shall see below in Sect. 2.4, our approach constructs a suitable colouring of $\tilde{J}$.
} 
Otherwise, we proceed as follows. The children of $y_{i}$ will be the elements of $\tilde{J}$ which are not already in the tree, and which are the reasons that we may not change the colour of $y_{i}$ without the colouring becoming $m$-unbounded or at least in danger of becoming so. Specifically, for each colour $j$ different from $\kappa\left(y_{i}\right)^{4}$ let $L_{j}^{(i)}$ be the subset of $L_{j}$ consisting of the lines $l_{j}$ going through $y_{i}$ such that

$$
\left|\left\{x \in l_{j} \cap J: \kappa(x)=j\right\}\right|+\left|\left\{x \in l_{j} \cap T_{i-1} \cap J: \kappa(x) \neq j\right\}\right| \geq m .
$$

(Here and later we abuse notation and use $T_{i-1}$ also to denote the vertex set of the tree $T_{i-1}$.) If the collection $L_{j}^{(i)}$ is empty for some $j \neq \kappa\left(y_{i}\right)$ we say that the colouring is advanceable at step $i .{ }^{5}$ Then we stop the construction, and declare $T:=T_{i-1}$.

Otherwise, if the colouring is not advanceable at step $i$, we define for each $j \neq \kappa\left(y_{i}\right)$ the set $I_{j}^{(i)}$ which consists of all the points of $J$ of colour $j$ on any line from $L_{j}^{(i)}$, excluding those points which are already in the tree $T_{i-1}$. We let the tree $T_{i}$ be the tree $T_{i-1}$ with the points from $I_{j}^{(i)}$ for all $j \neq \kappa\left(y_{i}\right)$ added as vertices, specifically as children of $y_{i}$. The edges of $T_{i}$ are those of $T_{i-1}$ together with edges linking $y_{i}$ to each of its children. Note that no child has the same colour as its parent. ${ }^{6}$

We remark that it is possible that $I_{j}^{(i)}$ may be empty for some colour $j$ even though $L_{j}^{(i)}$ is non-empty. This is the case if all the points of colour $j$ in $J$ which lie on any line in $L_{j}^{(i)}$ are vertices of $T_{i-1}$. If all of the $I_{j}^{(i)}$ are empty we let $T_{i}:=T_{i-1}$.

If the colouring is not advanceable at any step then eventually the tree will become fully constructed. We call such a colouring non-advanceable.

\subsection{The First Stage in the Construction}

To fix ideas, let us run through the first stage of the construction. We have $T_{0}=\left\{y_{1}\right\}=$ $\left\{x_{0}\right\}$ and so there is a first element of $T_{0}$. For each $j \in\{1,2, \ldots, d\}$ we have that $L_{j}^{(1)}$ is the subset of $L_{j}$ consisting of the lines $l_{j}$ going through $y_{1}$ such that

$$
\left|\left\{x \in l_{j} \cap J: \kappa(x)=j\right\}\right| \geq m
$$

since the second term on the left-hand side of (7) is zero. Since $\kappa$ is $m$-bounded, (8) means that $\left|\left\{x \in l_{j} \cap J: \kappa(x)=j\right\}\right|=m$. Now either $L_{j}^{(1)}$ is empty for some colour $j$, or it is non-empty for all $j$.

\footnotetext{
${ }^{4}$ In the case $i=1$ this simply means all colours $j$. This understanding applies in several places below.

5 See Sect. 2.4 Claim 2(b) for the reason we use this terminology.

${ }^{6}$ Note also that the sequence of points $y_{1}, y_{2}, y_{3}, \ldots$ is dependent on the particular colouring $\kappa$ we are looking at, and two different colourings will give rise in general to two different sequences of points. We should strictly therefore label our sequence as $y_{1}(\kappa), y_{2}(\kappa), y_{3}(\kappa), \ldots$ in order to emphasise this point. However we choose not to do this in order that our subsequent notation which involves expressions such as $\kappa\left(y_{i}\right)$-which strictly would be written $\kappa\left(y_{i}(\kappa)\right)$ and means the colour that the colouring $\kappa$ gives to the $i$ 'th member of the construction of the tree for the colouring $\kappa$-should not become too unwieldy.
} 
(1) In the first case we have that $\kappa$ is advanceable at step 1, we stop the procedure and declare $T=T_{0}$. Note that in this case, if for a certain $j, L_{j}^{(1)}=\emptyset$, then for all $l_{j} \in L_{j}$ passing through $y_{1}$ we have

$$
\left|\left\{x \in l_{j} \cap J: \kappa(x)=j\right\}\right|<m .
$$

In this case we can simply assign the colour $j$ to $x_{0}$ and we are done. (This observation will be important below.)

(2) Otherwise, when $L_{j}^{(1)} \neq \varnothing$ for all $j$, we have

$$
I_{j}^{(1)}=\left\{x \in J: \kappa(x)=j \text { and } x \in l_{j} \text { for some } l_{j} \in L_{j}^{(1)}\right\}
$$

and we note that since every line in each $L_{j}^{(1)}$ has exactly $m$ members of $J$ of colour $j$ on it, each $I_{j}^{(1)}$ has at least $m$ members, and so $T_{1}$ will be a proper extension of $T_{0}$, and in particular will have a second member ready for the construction of $T_{2}$.

The construction of the tree $T$ depends on the colouring $\kappa$; when we wish to emphasise this we shall use the notation $T(\kappa)$ and likewise $I_{j}^{(i)}(\kappa)$ to highlight this dependence.

\subsection{A Strict Partial Ordering}

Now we turn to the definition of the strict partial ordering on $K$. Take $\kappa_{1}, \kappa_{2} \in K$ and construct the trees $T\left(\kappa_{1}\right)$ and $T\left(\kappa_{2}\right)$. We say that $\kappa_{1}$ is more advanced than $\kappa_{2}$ at level $i_{0}$ if

(i) $I_{j}^{(i)}\left(\kappa_{1}\right)=I_{j}^{(i)}\left(\kappa_{2}\right)$ for all $j \neq \kappa_{1}\left(y_{i}\right)^{7}$ and for all $i<i_{0}$;

(ii) $I_{j}^{\left(i_{0}\right)}\left(\kappa_{1}\right) \subseteq I_{j}^{\left(i_{0}\right)}\left(\kappa_{2}\right)$ for all $j \neq \kappa_{1}\left(y_{i_{0}}\right)$;

(iii) at least one of the inclusions in item (ii) is strict.

In other words, $\kappa_{1}$ is more advanced than $\kappa_{2}$ at level $i_{0}$ if the coloured trees $T_{i_{0}-1}\left(\kappa_{1}\right)$ and $T_{i_{0}-1}\left(\kappa_{2}\right)$ are identical, and $T_{i_{0}}\left(\kappa_{1}\right)$ is a proper coloured subtree of $T_{i_{0}}\left(\kappa_{2}\right){ }^{8}$ This means in particular that $y_{i}\left(\kappa_{1}\right)=y_{i}\left(\kappa_{2}\right)$ for all $i \leq i_{0}$.

We say that $\kappa_{1}$ is more advanced than $\kappa_{2}$ if there is a level $i_{0}$ such that $\kappa_{1}$ is more advanced than $\kappa_{2}$ at level $i_{0}$. Note that there can be at most one such level because of the requirement of a strict inclusion at level $i_{0}$. It is clear that the notion of being more advanced is a strict partial ordering on $K$.

\footnotetext{
7 Once again we emphasise that $\kappa_{1}\left(y_{i}\right)$ means $\kappa_{1}\left(y_{i}\left(\kappa_{1}\right)\right)$.

8 Note that this expresses the idea that the construction of the tree for $\kappa_{1}$ as in the previous subsection is closer to termination than that for $\kappa_{2}$; hence the terminology "more advanced".
} 


\subsection{A Trichotomy}

Now, for a general colouring $\kappa \in K$ there are three possibilities. It may be advanceable at step 1, it may be advanceable at some step $i>1$ or it may be non-advanceable. We will prove the following claim.

\section{Claim 2}

(a) If $\kappa$ is advanceable at step 1 then we can extend $\kappa$ to an $m$-boundeded colouring of $\tilde{J}$.

(b) If $\kappa$ is advanceable at some step $i>1$ then there is a colouring $\tilde{\kappa} \in K$ which is more advanced than $\kappa$.

(c) If $\kappa$ is non-advanceable then $|T(\kappa) \cap J| \geq C_{d}^{-d} m^{d}$.

We will establish Claim 2 below, but for now we note that Claim 1 follows immediately from it. Indeed, the hypothesis of Claim 1 is that $m>C_{d}|J|^{1 / d}$, so

$$
|T(\kappa) \cap J| \leq|J|<C_{d}^{-d} m^{d},
$$

meaning that under the hypothesis of Claim 1 the third alternative cannot hold for any $\kappa \in K$. So every $\kappa \in K$ must be advanceable at some step. If $\kappa$ is advanceable at step 1, part (a) of Claim 2 gives us what we want; if not, $\kappa$ will be advanceable at some step $i>1$ and there will be a $\tilde{\kappa} \in K$ which is more advanced than $\kappa$. Once again, the third alternative cannot hold for $\tilde{\kappa}$, if the first alternative holds we are happy, and if the second alternative holds we obtain a $\tilde{\tilde{\kappa}}$ which is more advanced than $\tilde{\kappa}$. We iterate this process. Since $K$ is finite, a maximally advanced element of $K$ must exist, meaning that at some point of the iteration the second alternative cannot hold, leaving us with only the first. In summary, if $m>C_{d}|J|^{1 / d}$, for every $\kappa \in K$ there is some $\tilde{\kappa} \in K$ which is more advanced than $\tilde{\kappa}$ and which is advanceable at step 1 . Hence there exists an $m$-bounded colouring of $\tilde{J}$ as required.

This procedure gives an algorithm for actually constructing an $m$-bounded colouring, see Algorithm 1.

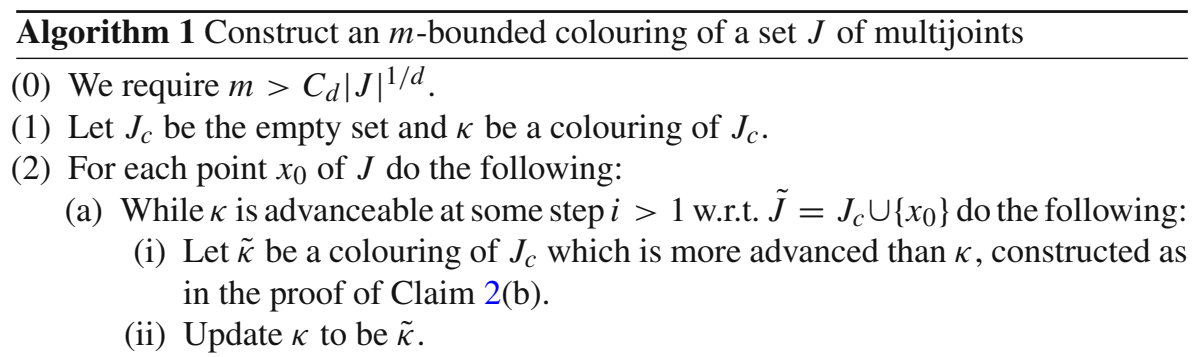

(b) Now $\kappa$ is advanceable at step 1.

(c) Extend $\kappa$ to $\tilde{J}$ by letting $\kappa\left(x_{0}\right)$ be some colour $j$ for which $L_{j}^{(1)}$ is empty.

(d) Update $J_{c}$ to be $\tilde{J}$.

(3) Now $\kappa$ is an $m$-bounded colouring of $J$. 
Note that we have already established case (a) of Claim 2 in the discussion of case (1) in Sect. 2.2. In the remainder of the proof we will verify the remaining two cases of Claim 2.

\subsection{Establishing Claim 2(b)}

For the second case of Claim 2, let us assume that $\kappa$ is advanceable at step $i_{0}>1$. That means that there is a colour $j_{0} \neq \kappa\left(y_{i_{0}}\right)$ such that for all lines $l_{j_{0}} \in L_{j_{0}}$ such that $y_{i_{0}} \in l_{j_{0}}$ we have

$$
\left|\left\{x \in l_{j_{0}} \cap J: \kappa(x)=j_{0}\right\}\right|+\left|\left\{x \in l_{j_{0}} \cap T_{i_{0}-1} \cap J: \kappa(x) \neq j_{0}\right\}\right|<m .
$$

We let $j_{1}=\kappa\left(y_{i_{0}}\right)$. Let us define a new colouring $\tilde{\kappa}$ which is identical to $\kappa$ except that $\tilde{\kappa}\left(y_{i_{0}}\right)=j_{0}$. What we shall show is that there is some $i_{1}<i_{0}$ such that $\tilde{\kappa}$ is more advanced than $\kappa$ at level $i_{1}$.

We first need to consider the effect of changing the colour of $y_{i_{0}}$ on the construction of the tree $T(\tilde{\kappa})$, and in particular, we need to bear in mind the possibility that $y_{i_{0}}$ might occur earlier in the construction of $T(\tilde{\kappa})$ than of $T(\kappa)$. As a rough guide, note that lines of colours other than the old and new colours of $y_{i_{0}}$ will play exactly the same role in both constructions, as will lines not containing $y_{i_{0}}$. We will need to examine vertices of the tree $T(\kappa)$ of colour either the old or new colour of $y_{i_{0}}$ for possible changes in the construction.

Specifically, we wish to verify that $\tilde{\kappa}$ is $m$-bounded and that $\tilde{\kappa}$ is more advanced than $\kappa$. The $m$-bounded conditions for $\tilde{\kappa}$ follow immediately from the corresponding conditions for $\kappa$ except for lines of colour $j_{0}$ which go through $y_{i_{0}}$. But for those lines we just saw that

$$
\left|\left\{x \in l_{j_{0}} \cap J: \kappa(x)=j_{0}\right\}\right|<m .
$$

and so

$$
\left|\left\{x \in l_{j_{0}} \cap J: \tilde{\kappa}(x)=j_{0}\right\}\right|=\left|\left\{x \in l_{j_{0}} \cap J: \kappa(x)=j_{0}\right\}\right|+1 \leq m .
$$

Thus $\tilde{\kappa}$ belongs to $K$ and it is meaningful to ask whether $\tilde{\kappa}$ is more advanced than $\kappa$. Since $y_{i_{0}} \in T(\kappa)$ we can find an index $i_{1}<i_{0}$ such that $y_{i_{0}}$ is a vertex of $T_{i_{1}}(\kappa)$ but not of $T_{i_{1}-1}(\kappa)$. That means that either $i_{1}=1$ or there exists a colour $j_{2} \neq j_{1}$ such that $\kappa\left(y_{i_{1}}\right)=j_{2}$ and a line $l_{j_{1}} \in L_{j_{1}}$ such that $y_{i_{0}}, y_{i_{1}} \in l_{j_{1}}$ and

$$
\left|\left\{x \in l_{j_{1}} \cap J: \kappa(x)=j_{1}\right\}\right|+\left|\left\{x \in l_{j_{1}} \cap T_{i_{1}-1} \cap J: \kappa(x) \neq j_{1}\right\}\right| \geq m .
$$

We want to show that $\tilde{\kappa}$ is more advanced than $\kappa$ at level $i_{1}$. First let us verify condition (i). If $i_{0}=1$ then condition (i) is vacuous. Otherwise it is clear that there are two types of steps we have to consider, and for other steps before $i_{1}$ condition (i) is immediate. The types of steps we have to consider correspond to vertices of the tree $T(\kappa)$ of colour equal to the new colour of $y_{i_{0}}$ and of colour equal to the old colour of $y_{i_{0}}$, and more precisely are: 
- steps $i_{2}$ such that $i_{2}<i_{1}$ and $\kappa\left(y_{i_{2}}\right) \neq j_{0}$ but there is a line $l_{j_{0}} \in L_{j_{0}}$ such that $y_{i_{2}}, y_{i_{0}} \in l_{j_{0}}$; and

- steps $i_{3}$ such that $i_{3}<i_{1}$ and $\kappa\left(y_{i_{3}}\right) \neq j_{1}$ but there is a line $\tilde{l}_{j_{1}} \in L_{j_{1}}$ such that $y_{i_{3}}, y_{i_{0}} \in \tilde{l}_{j_{1}}$.

For the former case we note that $y_{i_{0}} \notin T_{i_{2}}(\kappa)$ since this vertex set is a subset of $T_{i_{1}-1}(\kappa)$ which by assumption $y_{i_{0}}$ does not belong to. Therefore we see that as $i_{0}>i_{2}$

$$
\left|\left\{x \in l_{j_{0}} \cap T_{i_{0}-1} \cap J: \kappa(x) \neq j_{0}\right\}\right| \geq\left|\left\{x \in l_{j_{0}} \cap T_{i_{2}-1} \cap J: \kappa(x) \neq j_{0}\right\}\right|+1
$$

since $y_{i_{0}}$ is a member of the former set but not the latter. So (9) shows that

$$
\left|\left\{x \in l_{j_{0}} \cap J: \kappa(x)=j_{0}\right\}\right|+\left|\left\{x \in l_{j_{0}} \cap T_{i_{2}-1} \cap J: \kappa(x) \neq j_{0}\right\}\right|<m-1 .
$$

Now note that

$$
\left|\left\{x \in l_{j_{0}} \cap J: \tilde{\kappa}(x)=j_{0}\right\}\right|=\left|\left\{x \in l_{j_{0}} \cap J: \kappa(x)=j_{0}\right\}\right|+1
$$

and

$$
\left|\left\{x \in l_{j_{0}} \cap T_{i_{2}-1} \cap J: \tilde{\kappa}(x) \neq j_{0}\right\}\right|=\left|\left\{x \in l_{j_{0}} \cap T_{i_{2}-1} \cap J: \kappa(x) \neq j_{0}\right\}\right|,
$$

where the second equality follows since $\tilde{\kappa}$ and $\kappa$ are identical on $T_{i_{2}-1}$. So we obtain

$$
\left|\left\{x \in l_{j_{0}} \cap J: \tilde{\kappa}(x)=j_{0}\right\}\right|+\left|\left\{x \in l_{j_{0}} \cap T_{i_{2}-1} \cap J: \tilde{\kappa}(x) \neq j_{0}\right\}\right|<m
$$

and this shows that $l_{j_{0}} \notin L_{j_{0}}^{\left(i_{2}\right)}(\tilde{\kappa})$. Moreover, $l_{j_{0}} \notin L_{j_{0}}^{\left(i_{2}\right)}(\kappa)$ since if it were in this set then we would have $y_{i_{0}} \in T_{i_{2}}(\kappa)$ which is not possible as we saw above. So $y_{i_{0}}$ is not added to the vertices of $T(\tilde{\kappa})$ at step $i_{2}$, and we deduce that the iteration in the definition of the trees proceeds identically at this step for $\kappa$ and $\tilde{\kappa}$. That is, the coloured trees $T_{i_{2}}(\tilde{\kappa})$ and $T_{i_{2}}(\kappa)$ are identical.

For the latter case we note that $y_{i_{0}} \notin T_{i_{3}}(\kappa)$ since this vertex set is a subset of $T_{i_{1}-1}(\kappa)$ which by assumption $y_{i_{0}}$ does not belong to. That means that

$$
\left|\left\{x \in \tilde{l}_{j_{1}} \cap J: \kappa(x)=j_{1}\right\}\right|+\left|\left\{x \in \tilde{l}_{j_{1}} \cap T_{i_{3}-1} \cap J: \kappa(x) \neq j_{1}\right\}\right|<m .
$$

Now note that

$$
\left|\left\{x \in \tilde{l}_{j_{1}} \cap J: \tilde{\kappa}(x)=j_{1}\right\}\right|=\left|\left\{x \in \tilde{l}_{j_{1}} \cap J: \kappa(x)=j_{1}\right\}\right|-1
$$

and

$$
\left|\left\{x \in \tilde{l}_{j_{1}} \cap T_{i_{3}-1} \cap J: \tilde{\kappa}(x) \neq j_{1}\right\}\right|=\left|\left\{x \in \tilde{l}_{j_{1}} \cap T_{i_{3}-1} \cap J: \kappa(x) \neq j_{1}\right\}\right|,
$$

where the second equality follows since $\tilde{\kappa}$ and $\kappa$ are identical on $T_{i_{3}-1}$. Therefore

$$
\left|\left\{x \in \tilde{l}_{j_{1}} \cap J: \tilde{\kappa}(x)=j_{1}\right\}\right|+\left|\left\{x \in \tilde{l}_{j_{1}} \cap T_{i_{3}-1} \cap J: \tilde{\kappa}(x) \neq j_{1}\right\}\right|<m-1,
$$


and this shows that $\tilde{l}_{j_{1}} \notin L_{j_{1}}^{\left(i_{3}\right)}(\tilde{\kappa})$. Moreover, $\tilde{l}_{j_{1}} \notin L_{j_{1}}^{\left(i_{3}\right)}(\kappa)$ since if it were in this set then we would have $y_{i_{1}} \in T_{i_{3}}(\kappa)$ which is not possible as we saw above. So $y_{i_{0}}$ is not added to the vertices of $T(\tilde{\kappa})$ at step $i_{3}$, and we deduce that the iteration in the definition of the trees proceeds identically at this step for $\kappa$ and $\tilde{\kappa}$. That is, the coloured trees $T_{i_{3}}(\tilde{\kappa})$ and $T_{i_{3}}(\kappa)$ are identical.

Hence we conclude that the coloured trees $T_{i_{1}-1}(\tilde{\kappa})$ and $T_{i_{1}-1}(\kappa)$ are identical.

Now we verify conditions (ii) and (iii). We note that the only possible difference between the sets $L_{j}^{\left(i_{1}\right)}(\kappa)$ and $L_{j}^{\left(i_{1}\right)}(\tilde{\kappa})$ for some colour $j$ is that a line containing both $y_{i_{0}}$ and $y_{i_{1}}$ could be in one of these sets and not the other. We already know that the line joining these points is of colour $j_{1}$ so for other colours we have that $L_{j}^{\left(i_{1}\right)}(\kappa)$ and $L_{j}^{\left(i_{1}\right)}(\tilde{\kappa})$ are identical and so $I_{j}^{\left(i_{1}\right)}(\kappa)$ and $I_{j}^{\left(i_{1}\right)}(\tilde{\kappa})$ are identical too. For colour $j_{1}$ we have that $\kappa\left(y_{i_{0}}\right)=j_{1} \neq \tilde{\kappa}\left(y_{i_{0}}\right)$. This shows that $y_{i_{0}}$ is an element of $I_{j_{1}}^{\left(i_{1}\right)}(\kappa)$ but not of $I_{j_{1}}^{\left(i_{1}\right)}(\tilde{\kappa})$. Hence we conclude that the coloured tree $T_{i_{1}}(\tilde{\kappa})$ is a proper coloured subtree of $T_{i_{1}}(\kappa)$ and conditions (ii) and (iii) are verified.

This establishes the second case of Claim 2.

\subsection{Establishing Claim 2(c)}

For the last case of Claim 2, let us recall the statement of Quilodrán's lemma, which in [10] is proved for the case $\mathbb{F}=\mathbb{R}$. For the general case, see [2,5] or [11]. See also [9].

Lemma 1 Let $L$ be a collection of lines in $\mathbb{F}^{d}$ and let $J$ be a subset of the set of joints of L. Suppose that $J$ has the property that for every line $l \in L$ the cardinality of $l \cap J$ is either 0 or at least $m$. Then $|J| \geq C_{d} m^{d}$.

Let us then assume that $\kappa$ is non-advanceable. We let $\bar{J}$ be the set of points in $J$ which are vertices of the tree $T(\kappa)$. For a colour $j$ we let $\bar{L}_{j}$ be the union of the sets $L_{j}^{(i)}$ for those indices $i$ such that $\kappa\left(y_{i}\right) \neq j$. Then we let $\bar{L}=\bar{L}_{1} \cup \cdots \cup \bar{L}_{d}$.

We need to verify that the hypotheses of the lemma are satisfied for $\bar{J}$ and $\bar{L}$. First we note that the elements of $\bar{J}$ are in fact joints of $\bar{L}$. To see this, take $y_{i} \in \bar{J}$ with $i>1$ and assume that $\kappa\left(y_{i}\right)=j$. Then there is an $\tilde{\imath}<i$ and a line $l_{j} \in L_{j}^{(\tilde{l})}$ such that $y_{i} \in l_{j}$ and $\kappa\left(y_{i}\right) \neq j$ (since children always have a different colour from their parents). So $y_{i} \in \bar{L}_{j}$. Furthermore, for all colours $\tilde{j} \neq j$ we have by non-advanceability that the set $L_{\tilde{J}}^{(i)}$ is non-empty and the lines in these sets all go through $y_{i}$. So $y_{i} \in \bar{L}_{\tilde{\jmath}}$. Thus for each $j_{*} \in\{1, \ldots, d\}$, we have $y_{i} \in \bar{L}_{j_{*}} \subseteq L_{j_{*}}$. Since by hypothesis the collection $\mathcal{L}$ is generic, we conclude that $y_{i}$ is a joint of $\bar{L}$.

Now consider a line in $\bar{L}$, say $l_{j} \in L_{j}^{(i)}$. Then by definition of $L_{j}^{(i)}$ we have

$$
\left|\left\{x \in l_{j} \cap J: \kappa(x)=j\right\}\right|+\left|\left\{x \in l_{j} \cap T_{i-1} \cap J: \kappa(x) \neq j\right\}\right| \geq m .
$$

Note that all the points which are elements of the first of these sets will be vertices of $T_{i}$. Therefore the two sets occurring in this expression are subsets of $l_{j} \cap \bar{J}$ which 
are disjoint. Hence we have $\left|l_{j} \cap \bar{J}\right| \geq m$. This shows that all the hypotheses of the lemma are satisfied and so we deduce that $|\bar{J}| \geq C_{d}^{-d} m^{d}$.

Remark 5 The reader will observe that we use the hypothesis of genericity only in establishing Claim 2(c). We conjecture that the main result holds without this hypothesis.

Remark 6 We do not claim any particular efficiency for Algorithm 1. We simply wish to point out that our methods are constructive rather than existential.

Remark 7 It is natural to ask whether the method we have presented might be pushed in order to obtain the full multijoints bound (2). As noted above, the main difficulty is to replace the arithmetic mean bound $\sum_{j} S_{j}(x) \geq C_{d}$ for all $x \in J$ by the corresponding geometric mean bound $\prod_{j} S_{j}(x)^{1 / d} \geq C_{d}$. In fact it would suffice to obtain weighted arithmetic bounds $\sum_{j} \beta_{j} S_{j}(x) \geq C_{d}$ for all $x \in J$ for suitably chosen parameters $\beta_{j}$. Our methods, in conjunction with a suitable multiparameter version of Quilodrán's lemma, would seem to be applicable to this problem. We plan to return to this matter at a later date.

\section{References}

1. Bourgain, J., Guth, L.: Bounds on oscillatory integral operators based on multilinear estimates. Geom. Funct. Anal. 21(6), 1239-1295 (2011)

2. Carbery, A., Iliopoulou, M.: Counting joints in vector spaces over arbitrary fields (2014). arXiv: 1403.6438

3. Carbery, A., Valdimarsson, S.I.: The endpoint multilinear Kakeya theorem via the Borsuk-Ulam theorem. J. Funct. Anal. 264(7), 1643-1663 (2013)

4. Dvir, Z.: On the size of Kakeya sets in finite fields. J. Am. Math. Soc. 22(4), 1093-1097 (2009)

5. Dvir, Z.: Incidence theorems and their applications (2012). arXiv:1208.5073

6. Guth, L.: The endpoint case of the Bennett-Carbery-Tao multilinear Kakeya conjecture. Acta Math. 205(2), 263-286 (2010)

7. Guth, L., Katz, N.H.: Algebraic methods in discrete analogs of the Kakeya problem. Adv. Math. 225(5), 2828-2839 (2010)

8. Iliopoulou, M.: Personal Communication. IPAM (2014)

9. Kaplan, H., Sharir, M., Shustin, E.: On lines and joints. Discrete Comput. Geom. 44(4), 838-843 (2010)

10. Quilodrán, R.: The joints problem in $\mathbb{R}^{n}$. SIAM J. Discrete Math. 23(4), 2211-2213 (2009/2010)

11. Tao, T.: Algebraic combinatorial geometry: the polynomial method in arithmetic combinatorics, incidence combinatorics, and number theory. EMS Surv. Math. Sci. 1(1), 1-46 (2014) 